\title{
ON THE 5-MINUTE PHOTOSPHERIC OSCILLATION AND ITS MODELING
}

\author{
C. MARMOLINO ${ }^{1}$ and G. SEVERINO ${ }^{2}$ \\ ${ }^{1}$ Dipartimento di Scienze Fisiche dell'Università \\ Mostra d'Oltremare pad. 19 \\ I - 80125 Napoli, Italia \\ ${ }^{2}$ Osservatorio Astronomico di Capodimonte \\ Via Moiariello 16 \\ I - 80131 Napoli, Italia
}

\section{Introduction}

The 5-minute oscillations are standing waves trapped in subphotospheric cavities, whose evanescent part affect the profiles of photospheric lines.

The models developped to reproduce the signatures of these oscillations on spectral lines are not completely satisfactory. In fact

i) computed $I-V$ phases in very weak lines forming below $\mathrm{h} \sim 100 \mathrm{~km}$ are greater than those observed, $\geq 90^{\circ}$ against $\leq 60^{\circ}$ (e.g. Deubner, 1989);

ii) computed $I$ oscillations in the line wings are stronger than those in the line core, but the opposite is observed (Marmolino and Stebbins, 1989, their Fig. 3);

iii) computed and observed frequencies disagree significantly for higher degree modes which are confined in the very surface layers (Christensen-Dalsgaard et al., 1985).

To solve point iii) the use of an improved equation of state appears promising (Christensen-Dalsgaard et al., 1988). How this could help in the cases i) and ii) has not yet been investigated.

The theoretical results i) and ii) are based on an oscillation model which considers linear perturbations propagating in a compressible medium, assumed to be a perfect gas stably stratified, and damped by radiation according to the Spiegel's formula (Marmolino and Stebbins, 1989). The resulting vertical velocity $V$, temperature perturbation $T$ and pressure perturbation $P$ are, in an atmosphere characterized by a constant scale height $H$, sound velocity $c$, and radiative decay time $\tau_{r}$ :

$$
V \equiv v_{z}=v_{\circ} \exp \left(\frac{z}{h_{ \pm}}+i\left(\omega t-k_{x} x \mp k_{z} z\right)\right)
$$

J. O. Stenflo (ed.), Solar Photosphere: Structure, Convection, and Magnetic Fields, 251-254.

(C) 1990 by the IAU. 


$$
\begin{gathered}
T \equiv \frac{\delta T}{T}=\frac{(\gamma-1) \omega(1+i \alpha r)}{\left(\omega^{2}-c^{2} k_{x}^{2}\right)\left(1+\alpha^{2} r^{2}\right)}\left( \pm k_{z}+i\left(\frac{1}{h_{ \pm}}-\frac{c^{2} k_{x}^{2}}{\gamma \omega^{2} H}\right)\right) \cdot V \\
P \equiv \frac{\delta P}{P}=\frac{\gamma \omega(1+i \alpha r)}{\left(\omega^{2}-c^{2} k_{x}^{2}\right)\left(1+\alpha^{2} r^{2}\right)}\left( \pm k_{z}+\alpha\left(\frac{1}{h_{ \pm}}-\frac{1}{H}\right)+i\left(\frac{1}{h_{ \pm}}-\frac{1}{\gamma H} \mp \alpha k_{z}\right)\right) \cdot V
\end{gathered}
$$

with $h_{ \pm}=2 H /\left(1 \pm 2 H k_{i}\right), \alpha=1 /\left(\gamma \omega \tau_{r}\right), r=\left(\gamma \omega^{2}-c^{2} k_{x}^{2}\right) /\left(\omega^{2}-c^{2} k_{x}^{2}\right)$, and $k_{z}+i k_{i}$ the complex vertical wavenumber; in the double signs the upper and lower one corresponds to upward and downward phase propagation respectively.

In an effort to specify the origin of the discrepancies between theory and observations, we started a review of the model analysing its forecasts on a wider area of the $k-\omega$ diagram than that one where the 5-minute oscillations are confined. This work has revealed itself fruitful leading to the identification of well defined areas with different phase relations in the evanescent part of the $k-\omega$ diagram.

The preliminary results we present here refer to waves whose progressive part carries energy upwards all over the $k-\omega$ diagram.

\section{Results}

A contour map of the $T-V$ phase differences shows clearly the existence of two regimes in the evanescent part of the diagnostic diagram (Fig. 1b):

zone a, where $90^{\circ} \leq \phi_{T V} \leq 180^{\circ}$ and most of the 5 minute oscillations are confined; $\phi_{T V}=90^{\circ}$ without radiative damping;

zone $b$, where $\phi_{T V} \leq 0^{\circ} ; \phi_{T V}=-90^{\circ}$ without radiative damping.

The walls of zone $b$ are sharp and mark a jump of almost $\pm 180^{\circ}$ in the phase (exactly $180^{\circ}$ in the adiabatic case). Radiation damping fixes also the phase offset, $0^{\circ}$ with damping, $-90^{\circ}$ without. The walls coincide with the lines labelled $L_{m}$ and $f$ on the $k-\omega$ diagram in Fig. 1a, which have the equations $k_{x}^{2}=\omega^{2} / c^{2} \cdot\left(\gamma^{2} \omega^{2} \tau_{r}^{2}+\gamma\right) /\left(\gamma^{2} \omega^{2} \tau_{r}^{2}+1\right)$ and $\omega^{2}=g k_{x}$, respectively, where $g$ is the solar surface gravity.

The equation for $L_{m}$ is the real part of the dispersion relation for pure acoustic waves in the presence of radiative damping (e.g. Mihalas and Mihalas, 1984 their Eq. 101.11). On this line the denominator of the Eqs. (2) and (3) gets its minimum values and the topology of the surface representing the $T$ and $P$ amplitudes relative to $V$ over the $k-\omega$ diagram is dominated by the peaks occuring along it (Fig. 1c for the $\mathrm{T}$ perturbation). In absence of damping the denominator of Eqs. (2) and (3) is zero, reduces to the dispersion relation of $L a m b$ waves (curve $L$ in Fig. 1a), and corresponds to vanishing vertical velocity.

The equation for $f$ defines the fundamental mode. On this line and for the waves carrying energy upward the $T$ amplitude vanishes for $k_{x} \leq 1 / 2 H$ (Eq. 2). This behaviour is clearly seen both in the $T-V$ phase jump and in the contour maps involving the $T$ amplitude (Figs. 1c,d). 
The differentiation between the two evanescent zones is lost in the $P-V$ phase differences, since the phase jump on the fundamental mode disappears and $\phi_{P V} \sim-90^{\circ}$ with and without damping in both the zones.

The evanescent area at $k_{x} \leq 1 / 2 H$ is also characterized by a general increase of the amplitude of $P$ relative to $T$. This relative increase is strengthened by radiation damping which reduces significantly the $T$ amplitudes and smooths only $P$ amplitudes.
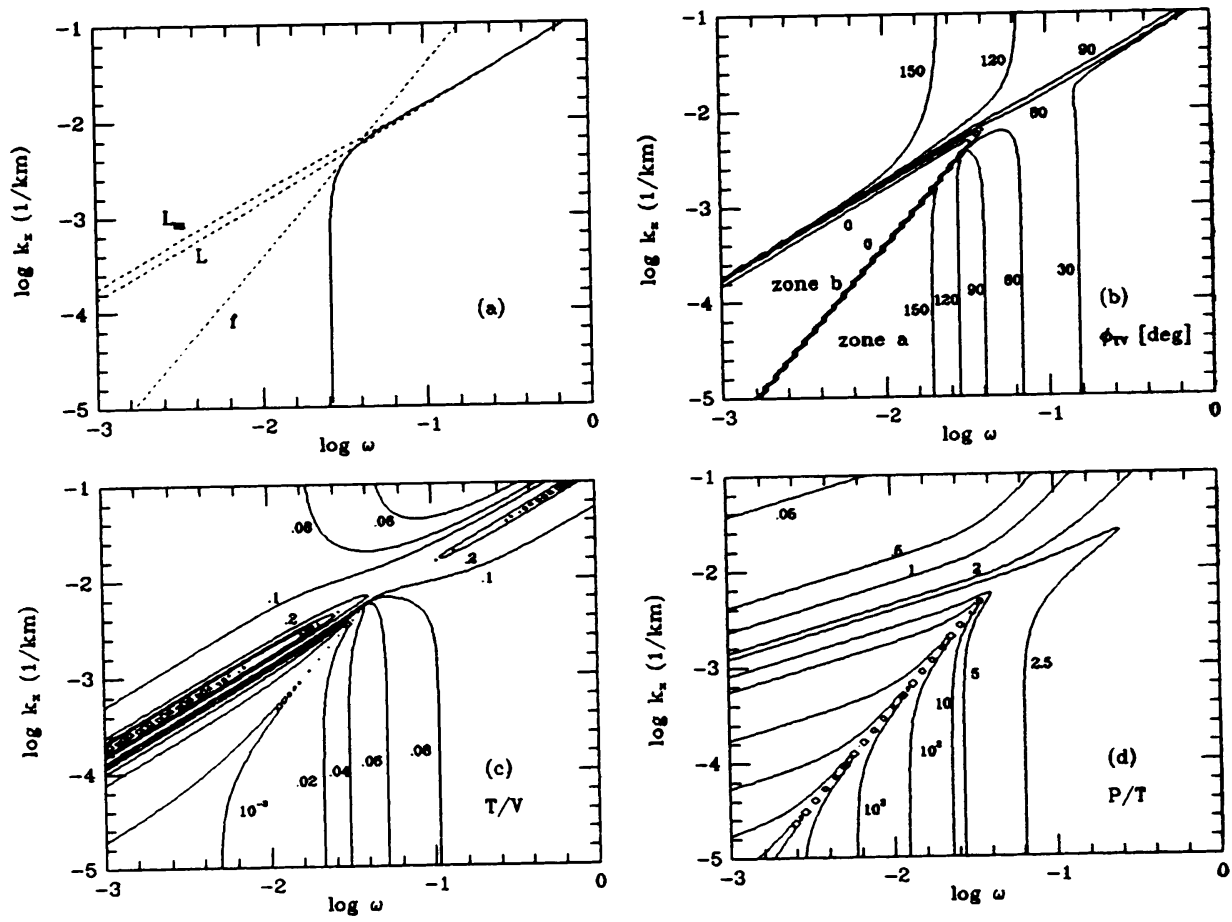

Figure 1. window (a): k- $\omega$ diagram for $T=4900^{\circ} K$, in presence of radiative damping, $\tau_{r}=15 s$. Solid line corresponds to $k_{z}^{2}-k_{i}^{2}=0$; dashed lines are the fundamental mode, labelled $f$, the modified Lamb waves, $L_{m}$, and the adiabatic Lamb waves, L. window (b): contour map of the $T-V$ phase differences; window (c): contour map of the $T$ amplitude relative to $V$; window (d): contour map of the $P$ amplitude relative to $T$.

\section{Conclusions}

Analysing the forecasts of the photospheric oscillation model on the $k-\omega$ diagram we found that the Lamb waves appear to be modified by the radiative damping, and that these modified Lamb waves and the fundamental mode are dividing lines in the phase relations between the different perturbations. Along these lines the vertical velocity and 
the temperature perturbation get their minima respectively.

The transition between zone $a$, of the ordinary 5 minute modes, and zone $b$, confined between fundamental mode and Lamb waves, has probably already been observed in the phase spectra of Schmieder (1976), Lites and Chipman (1979), Staiger (1984), and Deubner and Fleck (1989).

Pressures higher than temperatures are a possible source of discrepancy between theory and observation for the 5-minute modes. In fact since each perturbation has an amplitude increasing with height, oscillations stronger in the line wings than in the line core, as provided by theory, should imply core oscillations leaded by temperature and wing oscillations leaded by pressure.

We are completing this work considering waves whose progressive part carries energy downward. The model has to be improved allowing for complex horizontal wavenumber to define properly the modified Lamb waves. Finally, we plan to compute theoretical phase spectra for specific lines, which are directly comparable with observations, including the atmospheric temperature stratification and transfer effects.

\section{References}

Christensen-Dalsgaard, J., Gough, D. O. and Toomre, J.: 1985, Science, 229, 923

Christensen-Dalsgaard, J., Dappen, W. and Le Breton, J.: 1988, Nature, 336, 634

Deubner, F.-L.: 1989, this conference

Deubner, F.-L. and Fleck, B.: 1989, Astron. Astrophys., in press

Lites, B.W. and Chipman, E.G.: 1979, Astrophys. J., 231, 570

Marmolino, C. and Stebbins R. T.: 1989, Solar Phys., submitted

Mihalas, D. and Mihalas, D.W.: 1984, Foundations of Radiation Hydrodynamics, Oxford University Press, New York

Schmieder, B.: 1976, Solar Phys. 47, 435

Staiger, J., Schmieder, B., Deubner, F.-L. and Mattig, W.: 1984, Mem. Soc. Astron. Ital., 55, 147 